\title{
IMMERSIVE VISUALISATIONS IN DESIGN: USING AUGMENTED REALITY (AR) FOR INFORMATION PRESENTATION
}

\author{
A. Bravo ${ }^{凶}$ and A. M. Maier \\ DTU-Technical University of Denmark, Denmark \\ $\bigotimes$ abravo@dtu.dk
}

\begin{abstract}
Immersive visualisations introduce new possibilities for experiencing design, and as such for presenting information. To date, studies in design have focused mostly on immersive visualisations supporting product decisions. However, little attention has been paid to information presentation, e.g. in design reviews, for decisions in the boardroom, and/or for client presentations. This study with industry practitioners identifies information presentation practices and challenges, develops an immersive visualisation prototype, and explores opportunities for the use of immersive visualisations.
\end{abstract}

Keywords: communication, augmented reality (AR), virtual reality (VR), information presentation, immersive visualisation

\section{Introduction}

Recent advances in and widespread availability of immersive technologies, including technologies such as augmented and virtual reality (AR/VR), large screen display systems, and tangible user interfaces (TUIs) (e.g. Ishii and Ullmer, 1997), have enabled new ways of experiencing data, new ways of exploring, understanding, interacting with, and presenting information. This paper aims at exploring situations of presenting financial data in AR, following the virtuality continuum (Milgram and Kishino, 1994). We define immersive visualisations as information artefacts (Eckert et al., 2013) that make use of "engaging, embodied analysis tools to support data understanding and decision making" (Marriott et al., 2018). Examples of technologies used for such purposes include: large screen display systems (Cavallo et al., 2019), VR experiences to step into datasets (Millais et al., 2018), and data physicalisation with TUIs (Taher et al., 2015).

To date, studies in design have focused mostly on immersive visualisations for supporting product design decisions (Berg and Vance, 2016; Giunta et al., 2018; Jayaram et al., 2001; Liu and Boyle, 2009). However, little attention has been paid to its use for information presentation, e.g. during design reviews, for decisions in the boardroom, and/or for client presentations. Presentations are characterised as places for individuals to participate in decision making (Yoerger et al., 2015). Information presentation practices supporting decisions have for long been researched, e.g. in information sciences (Vessey, 1994), in management studies (Geraldi and Arlt, 2015; Meyer, 2000), and in data visualisation (Moore, 2017; Teets et al., 2010). However, research focusing specifically on the opportunities and challenges of using immersive visualisations in presentations is scarce. As such, this paper focuses on the presentation of financial information in AR for decision support, aiming to: (1) identify current information presentation practices and challenges for decision 
support, (2) identify opportunities for using immersive visualisations addressing the challenges previously identified, and (3) review experiences of using one example of an immersive visualisation developed during this study.

The remainder of the paper is structured as follows. Section 2 provides a literature background, bringing together state-of-the art from information presentation and visualisations (2.1) and research on immersive visualisations in design presented according to the type of technology used, namely AR, VR, and TUIs (2.2). In Section 3, an overview of the interview- and workshop-based study with industry representatives is given. Section 4 outlines the results, limitations of the study and implications for design research and practice. Section 5 presents the conclusions of the study and avenues for further work.

\section{Literature background}

\subsection{Information presentation and visualisations}

Despite an abundance of tools and technologies for information presentation, there are no commonly accepted guidelines that describe the optimal manner of presenting data (Meyer, 2000). Literature reviews have shown conflicting results due to the lack of a consistent methodology for the assessment of information presentation techniques with different types of displays (Coll and Coll, 1993; Jarvenpaa et al., 1984). In addition, optimal information presentation is dependent on a number of variables that include the type of display and the task-at-hand (Meyer et al., 1997).

Effective visualisations create a bridge between the quantitative nature of data and human intuition (Donalek et al., 2014). The ability for knowledge discovery and understanding through visualisations critically depends on how the data is presented. Cognitive fit theory explains the cognitive aspects implied in the use of tables (symbolic representations) and the use of visualisations (spatial representations) for presenting data (Vessey, 1994). Depending on the use case, one representation may be more adequate than another. As data complexity increases, the decision maker has more information to acquire and evaluate to make the decision, potentially leading to an increase in cognitive load (Wood, 1986). Visualising data appropriately is said to lower cognitive load and thereby facilitate decision making (Wickens and Carswell, 1995).

What might be an appropriate data visualisation? There are three types of data visualisations according to Cairo (2019): exploratory, experiential and explanatory. (1) Exploratory visualisations enable looking into the data in order to help the readers "explore" and find their own insights in the dataset. This type of visualisation is central to data visualisation with immersive technologies. Its aim is to improve understanding of complex datasets and multidimensional data (Donalek et al., 2014; Millais et al., 2018). (2) Experiential visualisations create an emotion in the reader and thus lead to action. (3) Explanatory visualisations are about articulating insights found in the data to an audience. This type is most common in presentation settings: the data is prepared and analysed for the decision maker and usually displayed with static visual content. Here, we inquire further into the use of explanatory visualisations with AR.

The use of immersive visualisations opens the field for multisensory display of information that makes use of the sense of touch and proprioception - the perception of awareness of the position and movement of the body - to experience data in an intuitive and engaging manner, i.e. with tangible interaction and force feedback (Nakagaki et al., 2019). It has further been stated that immersion in information presentation enhances engagement in data-informed decision making and supports social engagement in collaborative settings (Chandler et al., 2015; Marriott et al., 2018).

\subsection{Augmented reality, virtual reality, and tangible user interfaces in design}

The adoption of immersive technologies in design has been found to facilitate shared understanding in the design project (Hoang et al., 2019). On the one hand, VR has raised the interest of engineering design for two core reasons: (1) the possibility to deploy 3D models in a 3D space to foster design decisions (Berg and Vance, 2016; Jayaram et al., 2001) and (2) usability testing of products before proceeding to physical prototyping (Liu and Boyle, 2009). On the other hand, concerning AR 
research for design, a study revealed that immersive visualisations are mostly researched for the "preliminary layout", "definitive layout", and "concept" phases in design (Giunta et al., 2018). For example, the use of immersive visualisations for design representations with spatial augmented reality (O'Hare et al., 2018).

Design activities are embodied, situated and multi-modal (Comi et al., 2019). Through multisensory input and output devices, immersive visualisations enable to 'step into the data' and to interact with data using your whole body. This is facilitated by devices such as TUIs that enable interacting with gestures and different parts of the body. Previous studies show that TUIs promote communication among participants during collaborative design processes (Shamonsky and Mitchell, 2003) and suggest their potential use in situations of distributed decision making (Ratti et al., 2004).

Kim and Mahler (2008) evaluated immersion in design by comparing the same task conducted with a TUI in the form of a tabletop system with interactive 3D blocks and a graphical user interface (GUI). Their results suggest that immersion with TUIs aids to structure a designer's spatial cognition and that higher degrees of immersion provide higher degrees of spatial understanding (Bowman and McMahan, 2007) - a result that has been corroborated in a study on spatial perception of products with varying complexity levels using VR as the immersive technology (Horvat et al., 2019).

\section{Study methods}

The aim of the explorative study reported in this paper was to get an initial understanding on information presentation practices, challenges, and opportunities for using immersive, explanatory visualisations in presentation settings. This study is the starting point of a longer-term collaboration with a company specialised in the design and development of virtual- and augmented reality solutions. In this paper, we present the first steps of researching and developing an immersive visualisation of financial data using HoloLens (Microsoft, 2016), with the overall aim to support decision making in presentation settings.

HoloLens is an AR device that presented two major advantages for our study: (1) the inclusion of the real-world environment and other participants in the experience given that presentations are collective activities in a situated context. (2) A study on immersive technologies showed HoloLens in the lead for highly interactive tasks compared to the use of tablet AR (Bach et al., 2018).

The study consisted of three phases outlined in Figure 1. The first phase included seven interviews with industry practitioners working with data and information presentation. The objective was to get an initial understanding of the current practices and challenges in the presentation of information as well as the opportunities for using immersive visualisations in presentation settings. The second phase included the design, development, and testing of the first and second iterations of a prototype based on data obtained from the industry collaborator. The third phase consisted of conducting a workshop where a group of industry practitioners tested the immersive visualisation developed.

Table 1 lists the 11 participants of the study. Industry participants include both data analysts and managers/executives. Interviews and workshop were conducted at the respective companies.

Table 1. Research methods and study participants during interview and workshop

\begin{tabular}{|c|c|c|c|}
\hline \multirow{2}{*}{ Research method } & \multicolumn{2}{|c|}{ Study participants' role } & \multirow{2}{*}{ Company information } \\
\cline { 2 - 3 } & Data analyst & Manager / Executive & \\
\hline Interview & 2 & 1 & Business and management consultancy \\
& 1 & 1 & Large accounting and auditing consultancy \\
& - & 1 & Digitalisation consultancy \\
& - & 1 & Large telecommunications company \\
\hline Workshop & 2 & 2 & Large accounting and auditing consultancy \\
\hline
\end{tabular}




\section{INTERVIEWS}

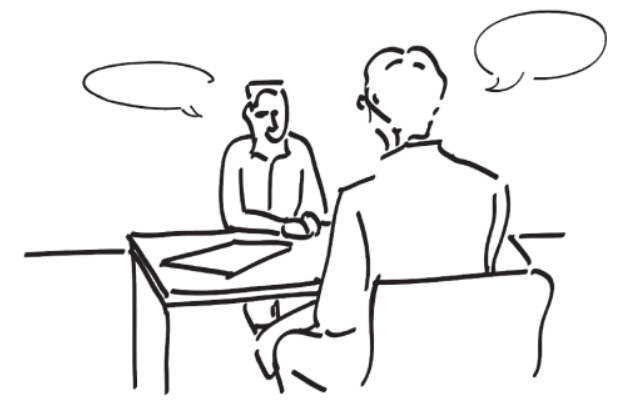

Seven interviews with industry participants to explore current practices and challenges in information presentation and to explore opportunities for the use of immersive visualisations.

\section{Research questions:}

1. What are current information presentation practices used for decision support?

2. What are the challenges in current information presentation practices for decision support?

3. What are the opportunities for using immersive visualisations in presentations?

\section{PROTOTYPE DESIGN- AND DEVELOPMENT PROCESS}

1. Concept design of an immersive visualisation based on

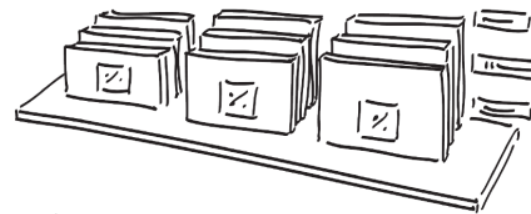

Immersive Visualisation: Stacked Area Chart financial valuation data.

2. Design and development together with collaborating AR development company.

3. Internal-testing of the immersive visualisations prototype with AR development company.

The final design is a stacked area chart as immersive visualisation in AR displaying financial valuation data.

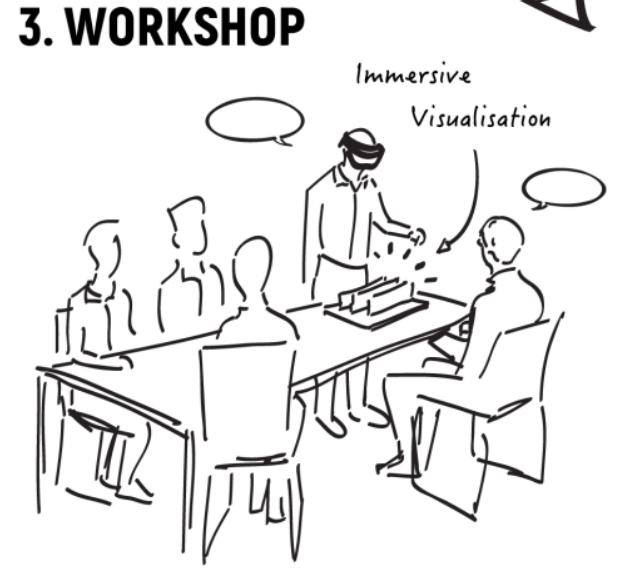

Workshop participants for first evaluation of the immersive visualisation prototype developed.

Workshop structure:

1. Initial 10-minute training on how to use Microsoft HoloLens.

2. Individual exploration using Microsoft HoloLens of the developed immersive visualisation. Simultaneously, the researcher asked the study workshop participant wearing the headset about the experience of using an immersive visualisation for the presentation of - in this study - financial valuation data.

3. Collective review of the immersive visualisation.

Figure 1. Study phases

\subsection{Interviews}

The goal of the interviews was to assess the current situation of presentation practices and challenges in industry. Furthermore, we inquired about the opportunities for using immersive visualisations in presentation settings. The interviews lasted between one hour and an hour and a 
half and were recorded with permission of the participants. The interview protocol consisted of open-ended questions aiming to answer the following research questions (RQs):

1. What are current information presentation practices used for decision support?

2. What are the challenges in current information presentation practices for decision support?

3. What are the opportunities for using immersive visualisations in presentations?

Interviews were transcribed verbatim and the data was coded using Atlas.ti 8. The first step consisted of the identification of parts of the conversation directly related to the following topics: (1) information presentation practices, (2) challenges, and (3) opportunities for using immersive visualisations in Microsoft HoloLens. Answers were then grouped into categories and duplicates were eliminated through affinity diagramming. The results are categorised in Figure 3 (section 4.1).

\subsection{Prototype design and development}

Reporting on the whole design and development process is outside the scope of this paper. For the purpose of this paper, the visualisation was developed with our industry collaborator and displayed a statistical graph representing valuation data, which is financial data calculated to determine the present value of a company (Figure 2).

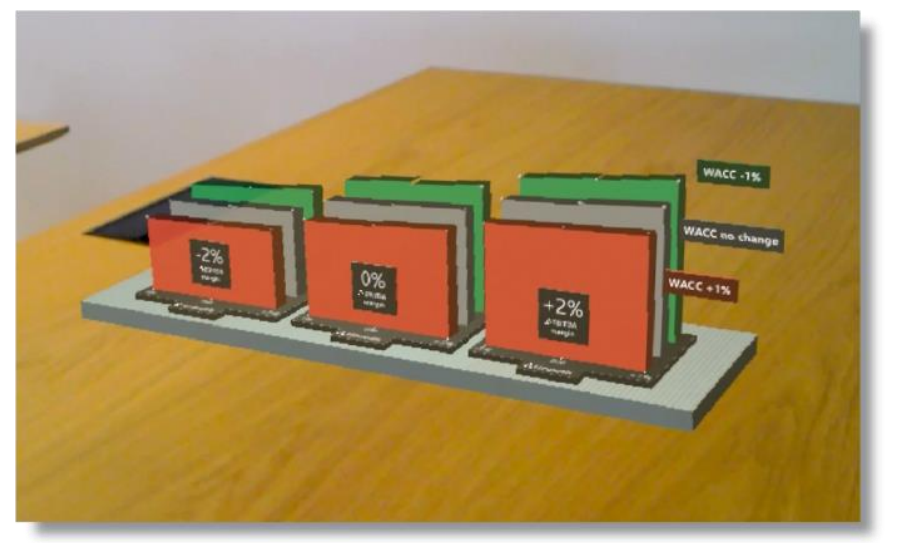

Figure 2. Immersive visualisation as 3D stacked area charts used in the workshop

The visualisation was designed to optimise perception in AR. For example, through the use of numerical cues appearing above data points upon directing the gaze towards them and a clear labelling of the interface. The interactive possibilities allowed for a gradual change in one of the variables of the visualisation through an interactive menu. The visualisation was intended to be displayed in front of each participant and positioned at the centre of the table with the same angle with respect to each participant. The centre of the table was chosen to reproduce a typical location around which meeting participants present information.

\subsection{Workshop}

The aim was to assess the experience of using an immersive visualisation for the presentation of data. We chose a workshop setting to have participants practically experience the immersive visualisation. The workshop lasted two hours and consisted of four participants, some of whom had already participated in the prior interview study.

The workshop protocol consisted of an initial 10-minute training on how to use Microsoft HoloLens, including training in basic gestures and interaction modalities presented by the system. Following the training, each participant wore the headset in turns to explore the capabilities of the immersive visualisation displayed for its use in presentations. Alongside the process of exploration, the interviewer asked each participant about the experience of using the immersive visualisation for decision support. When all participants had worn the headset, we held a collective review on the uses of immersive visualisations for presentations. 
The workshop was audio recorded with the permission of the participants. An overall feedback presentation of the study results was given to the main company collaborator for further input into the development of the prototype. The next section reports and discusses the results obtained.

\section{Study results}

The approach used in this explorative study provided an initial answer to our questions on presentation practices and challenges for decision support. Furthermore, we inquired into opportunities for using an immersive visualisation in AR for the presentation of information.

\subsection{Information presentation practices, challenges, and opportunities}

Analysing the data, for each of the three categories (current practices, challenges, opportunities), multiple themes are identified and illustrated in Figure 3. Each category corresponds to one of the research questions presented in section 3.1.

- Information presentation practices for decision support. We observe that information presentation practices minimise cognitive load by reducing the amount of information presented. Information is usually presented with text and static visualisations. Complex information is presented in blocks to avoid cognitive load. One interviewee told us about a 5second rule prescribing that tables and visualisations should be designed to be understood in 5 seconds or less. Another interviewee added: "Reduce the number of graphs so there is one simple message." The priority is clarity in the message by conveying one simple relevant message. As one interviewee said: "If you want to present something for decision making, it should be clear, it should be obvious." Favouring shared understanding in presentations is key. In order to achieve so, a common practice is to use knowledge, terminology, and visualisations familiar to decision makers.

- Challenges in information presentation practices for decision support. Complex information presents a challenge in presentations. One interviewee commented: "You need to put in a real effort to make it simple enough to explain and make people understand." Another challenge emphasises showing numbers in tables or visualisations without displaying the context of the data. As one interviewee reported: "The biggest issue I have seen on board level, basically anywhere, is to add context to the data." The interviewee followed-up explaining that this could lead to misunderstandings in assessing a decision that could then lead to a wrong decision, because of the lack of understanding of the context.

- Opportunities for using immersive visualisations in presentations. Participants commented that immersive visualisations offer the possibility simultaneously to perceive the data (virtual stimulus) and the participants involved in the presentation (real-world stimulus) in an AR environment. Furthermore, the 3D space allows to map spatial data in the real-world, i.e. to visualise the impact of an on-shore wind turbine park in the environment. Some interviewees mentioned that the 3D space could also be used to add an extra dimension to statistical data. This case, however, would require further study in presentation settings as it might increase the complexity of the given representation. Further, immersive visualisations could enable a gradual display of information, i.e. with the use of animations. This last case is envisioned by one interviewee: "Instead of having a complicated slide that nobody really understands [...]; if you could pull one number at a time, in real-time, and then put together the blocks so that decision makers understand what I am describing while I am doing it; I think the way of presenting and understanding my methodology [...] would be much better." In addition, realtime input of information could be incorporated into the presentation to include the decision makers" feedback in the visualisations presented. As one interviewee commented: "Real-time working with numbers would be highly relevant."

Interview results also point to considerations concerning additional time that needs to be allocated for an initial training with the technology, the extra cost of using the hardware and software, and the extra time that its use may imply during the presentation. 


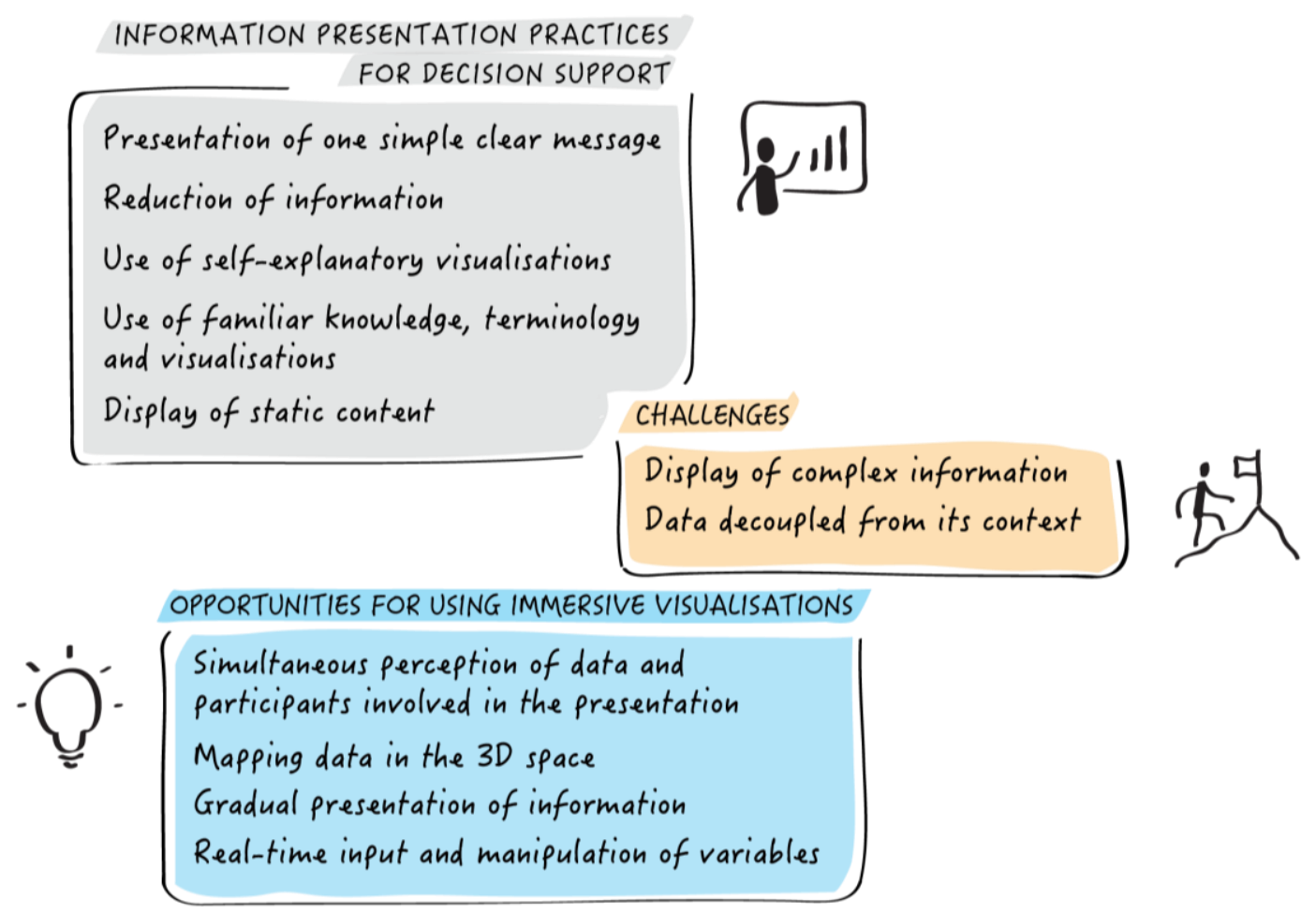

Figure 3. Themes elicited from interview transcripts

\subsection{Immersive visualisations: prototype use}

The objective of the workshop was to assess the experience of using an immersive visualisation in presentations. For this, a HoloLens prototype for presenting financial data was designed and developed. The following paragraphs describe the themes that emerged during the exploration of the prototype:

- Presence and engagement. As one of the interviewees noted, immersive visualisations involved "being present and engaging [with data]." Another participant commented on the benefit of presenting data and being able to perceive both the participants and the data in the same space around the table, "instead of turning around looking at a screen or pointing on a computer."

- Accessibility of information for non-data experts. Participants in presentations may not be data experts, therefore immersive visualisations could facilitate the understanding of the information presented. One participant explained: "It should be much easier to understand for our typical client, who is not used to talking about [financial data]."

- Enhanced interaction coupled to the presentation narrative. All participants agreed that immersive visualisations provided enhanced interaction capabilities in comparison to the slide presentations they used. One participant suggested that the interactive possibilities of immersive visualisations could enable "to build visualisations as you speak." Another participant commented on the use of immersive visualisations during the presentation as building blocks "like Lego", to make communication easier and more understandable.

- Directed focus of attention coupled to the presentation narrative. One participant commented on the usefulness of introducing numbers at the right moment along the presentation narrative, in order to direct the focus of attention of the decision maker. The following comment illustrates the situation: "You have 30 numbers and despite that we start talking about one of them, the eyes [of the decision makers] are fluctuating around the 29 others."

- Real-time input of data. To visualise the change of specific variables during the presentation. For example, one participant explained: "In reality we have planned 95\% [of the meeting]. But we would like to have the client input and we have a margin of 5\% that we would like to switch around in the meeting. [...] We would like to make it more dynamic to get the clients' input into the conclusions." 
- Invasiveness of immersive visualisations. Participants reported that using a headset would very likely alienate the relationships between individuals during presentations. The novelty of the display and visualisation might distract decision makers from the content of the presentation.

Overall, these results suggest that immersive visualisations engage and bring interactive possibilities for the presentation of information that can be coupled with the narrative of the presentation. However, immersive visualisations should be used mindfully to avoid presenting eye-appealing information that distract the decision maker from the content presented.

\subsection{Limitations of the study}

Using a qualitative interview- and workshop study was deemed appropriate for this initial investigation. However, research at this stage cannot claim causal links between the use of immersive visualisations in AR and the quality of decision support. Further, results of the study have to be read within the context of immersive visualisation of financial data developed using Microsoft HoloLens.

\subsection{Implications for design research and practice}

The results from the interviews and the workshop present a common vision towards: (1) simultaneous perception of data and participants in the presentation space and (2) interactivity and engagement provided by immersive visualisations. Contrary to our expectations, participants did not search for novel presentation methods to support decisions but rather sought for a familiar display of information. This finding is consistent with theory stating that confidence in decisions is related to the familiarity of the visual representation of the problem (Geraldi and Arlt, 2015).

Interactivity coupled with the narrative of the presenter, displayed gradually as blocks of information to facilitate understanding, opens the field for an in-depth exploration of "fluidity" in immersive visualisations. The notion of fluidity refers to the transformation of visual objects from "frozen" (static) to "fluid" (dynamic) across the unfolding practice of design (Whyte et al., 2007). Fluidity through immersive visualisations may generate new ways of exchanging knowledge and negotiating meaning in design sessions. Designing can be considered a "reflective conversation with the materials of the situation" (Schön, 2003) and reinforced interactivity could enhance the reflection-in-action process of designers. Other applications could envisage co-creative sessions in design: the designer makes a "move" that causes changes in the immersive visualisation displayed. Immersive visualisations would become "fluid" and transform from static to dynamic, dynamic to static, in a rhythm paced by the evolution of the designers' constructs.

\section{Summdary and conclusions}

This paper describes an exploratory study on the presentation of financial information in augmented reality (AR) for decision support. Through seven interviews, we have identified: (1) current information presentation practices, highlighting that a simple message is key for presentations aiming to support decisions; (2) Challenges in information presentation practices consisting of the presentation of complex information and the contextualisation of data; (3) Opportunities for the use of immersive visualisations consisting of simultaneous perception of participants and data in AR environments which includes mapping data in the 3D space, gradually presenting information, and dynamically inputting data during the presentation. The workshop results have shown that immersive visualisations engage participants with the data presented and enable interactive presentation of information that, coupled with the narrative of the presenter, may enhance communication and decision support.

Reflecting on the results both from the interviews and the workshop, the main takeaway are the opportunities for interacting with information overlaid onto the real world during information presentation. This includes directing the focus of attention of participants through interactive elements presented alongside the unfolding of the presentation narrative. Although interactivity is not exclusive to immersive visualisations and can also be achieved through data visualisation tools displayed on a screen or projected onto a surface, what emerged in this exploratory study are the interactive possibilities that 
simultaneous perception of digital information and fellow participants in the same physical space bring to information presentation. In addition, participants in the workshop reported that with an immersive visualisation in AR, they felt more engaged during the presentation compared to their usual presentation settings. The phenomenon of coupling digital information to the environment is particular to immersive visualisations in AR and warrants further research attention in design.

\section{Acknowledgements}

Funding has been received from the European Union (EU) Horizon 2020 Framework Programme for Research and Innovation under Grant Agreement No. 770420 - EURITO. The authors would also like to thank the industry collaborator Virsabi ApS for facilitating the recruitment of study participants, participating in the data collection, and for the development of the immersive visualisation in AR.

\section{References}

Bach, B. et al. (2018), "The Hologram in My Hand: How Effective is Interactive Exploration of 3D Visualizations in Immersive Tangible Augmented Reality?", IEEE Transactions on Visualization and Computer Graphics, Vol. 24 No. 1, pp. 457-467. https://doi.org/10.1109/TVCG.2017.2745941

Berg, L.P. and Vance, J.M. (2016), “An Industry Case Study: Investigating Early Design Decision Making in Virtual Reality”, Journal of Computing and Information Science in Engineering, Vol. 17 No. 1, pp. 011001. https://doi.org/10.1115/1.4034267

Bowman, D.A. and McMahan, R.P. (2007), "Virtual reality: How much immersion is enough?”, Computer, Vol. 40 No. 7, pp. 36-43. https://doi.org/10.1109/MC.2007.257

Cairo, A. (2019), "The functional art: an introduction to information graphics and visualization", The Functional Art, Musings on the Modes of Visualization Design. https://doi.org/10.5860/choice.50-3652

Cavallo, M. et al. (2019), "Dataspace: A Reconfigurable Hybrid Reality Environment for Collaborative Information Analysis", IEEE Conference on Virtual Reality and 3D User Interfaces (VR), IEEE, pp. 145153. https://doi.org/10.1109/VR.2019.8797733

Chandler, T. et al. (2015), "Immersive Analytics", Big Data Visual Analytics, BDVA 2015, IEEE, pp. 529-533. https://doi.org/10.1145/2992154.2996365

Coll, J.H. and Coll, R. (1993), "Tables and graphs: A classification scheme for display presentation variables and a framework for research in this area", Information Processing and Management, Vol. 29 No. 6, pp. 745-750. https://doi.org/10.1016/0306-4573(93)90103-K

Comi, A., Jaradat, S. and Whyte, J. (2019), "Constructing shared professional vision in design work: The role of visual objects and their material mediation”, Design Studies, Elsevier Ltd, Vol. 64 No. 1994, pp. 90-123. https://doi.org/10.1016/j.destud.2019.06.003

Donalek, C. et al. (2014), "Immersive and collaborative data visualization using virtual reality platforms", IEEE International Conference on Big Data (Big Data), IEEE, pp. 609-614. https://doi.org/10.1109/BigData. 2014.7004282

Eckert, C., Stacey, M. and Earl, C. (2013), "Formality in design communication", Artificial Intelligence for Engineering Design, Analysis and Manufacturing: AIEDAM, Vol. 27 No. 2, pp. 91-103. https://doi.org/ $10.1017 / \mathrm{S} 0890060413000073$

Geraldi, J. and Arlt, M. (2015), Visuals Matter!, Project Management Institute, Pennsylvania.

Giunta, L. et al. (2018), "A Review of Augmented Reality Research for Design Practice: Looking to the Future", NordDesign, Vol. 2018

Hoang, D. et al. (2019), “Adopting Immersive Technologies for Design Practice: The Internal and External Barriers", Proceedings of the Design Society: International Conference on Engineering Design, Vol. 1 No. 1, pp. 1903-1912. https://doi.org/10.1017/dsi.2019.196

Horvat, N. et al. (2019), "Comparing Virtual Reality and Desktop Interface for Reviewing 3D CAD Models", Proceedings of the Design Society: International Conference on Engineering Design, Vol. 1 No. 1, pp. 1923-1932. https://doi.org/10.1017/dsi.2019.198

Ishii, H. and Ullmer, B. (1997), "Tangible bits", Proceedings of the SIGCHI Conference on Human Factors in Computing Systems - CHI '97, ACM Press, New York, New York, USA, pp. 234-241. https://doi.org/ $10.1145 / 258549.258715$

Jarvenpaa, S.L., Dickson, G.W. and DeSanctis, G. (1984), "Methodological issues in experimental IS research: experiences and recommendations", Proceedings of the International Conference on Information Systems, pp. 155-156. https://doi.org/10.2307/249115 
Jayaram, S. et al. (2001), "Assessment of vr technology and its applications to engineering problems", Journal of Computing and Information Science in Engineering, Vol. 1 No. 1, pp. 72-83. https://doi.org/10.1115/ 1.1353846

Kim, M.J. and Maher, M.L. (2008), "The impact of tangible user interfaces on spatial cognition during collaborative design", Design Studies, Vol. 29 No. 3, pp. 222-253. https://doi.org/10.1016/j.destud. 2007.12.006

Liu, S. and Boyle, I.M. (2009), "Engineering design: perspectives, challenges, and recent advances", Journal of Engineering Design, Vol. 20 No. 1, pp. 7-19. https://doi.org/10.1080/09544820802670914

Marriott, K. et al. (2018), Immersive Analytics State-of-the-Art, Vol. 11190, Springer International Publishing, https://doi.org/10.1007/978-3-030-01388-2

Meyer, J. (2000), "Performance with tables and graphs: Effects of training and a visual search model", Ergonomics, Vol. 43 No. 11, pp. 1840-1865. https://doi.org/10.1080/00140130050174509

Meyer, J., Shinar, D. and Leiser, D. (1997), "Multiple factors that determine performance with tables and graphs", Human Factors, Vol. 39 No. 2, pp. 268-286. https://doi.org/10.1518/001872097778543921

Microsoft (2016), "Microsoft HoloLens".

Milgram, P. and Kishino, F. (1994), “A Taxonomy of Mixed Reality Visual Displays”, IEICE Transactions on Information Systems, pp. 1321-1328.

Millais, P., Jones, S.L. and Kelly, R. (2018), "Exploring Data in Virtual Reality”, Extended Abstracts of the 2018 CHI Conference on Human Factors in Computing Systems - CHI '18, pp. 1-6. https://doi.org/10.1145/ 3170427.3188537

Moore, J. (2017), "Data visualization in support of executive decision making", Interdisciplinary Journal of Information, Knowledge, and Management, Vol. 12, pp. 125-138. https://doi.org/10.28945/3687

Nakagaki, K. et al. (2019), "Inforce: Bi-directional 'Force' Shape Display For Haptic Interaction”, TEI 2019 Proceedings of the 13th International Conference on Tangible, Embedded, and Embodied Interaction, pp. 615-623. https://doi.org/10.1145/3294109.3295621

O'Hare, J. et al. (2018), "Defining requirements for an Augmented Reality system to overcome the challenges of creating and using design representations in co-design sessions", CoDesign, Taylor \& Francis, Vol. 00 No. 00, pp. 1-24. https://doi.org/10.1080/15710882.2018.1546319

Ratti, C. et al. (2004), “Tangible User Interfaces (TUIs): A novel paradigm for GIS”, Transactions in GIS, Vol. 8 No. 4, pp. 407-421. https://doi.org/10.1111/j.1467-9671.2004.00193.x

Schön, D.A. (2003), The Reflective Practitioner: How Professionals Think in Action, Ashgate Publishing Limited.

Shamonsky, D.J. and Mitchell, W.J. (2003), Tactile, Spatial Interfaces for Computer-Aided Design, Massachusetts Institute of Technology.

Taher, F. et al. (2015), "Exploring interactions with physically dynamic bar charts", Conference on Human Factors in Computing Systems - Proceedings, Vol. 2015 No. April, pp. 3237-3246. https://doi.org/10.1145/ 2702123.2702604

Teets, J.M., Tegarden, D.P. and Russell, R.S. (2010), "Using cognitive fit theory to evaluate the effectiveness of information visualizations: An example using quality assurance data", IEEE Transactions on Visualization and Computer Graphics, IEEE, Vol. 16 No. 5, pp. 841-853. https://doi.org/10.1109/TVCG.2010.21

Vessey, I. (1994), "The effect of information presentation on decision making: A cost-benefit analysis", Information and Management, Vol. 27 No. 2, pp. 103-119. https://doi.org/10.1016/0378-7206(94)90010-8

Whyte, J.K. et al. (2007), "Visual practices and the objects used in design", Building Research and Information, Vol. 35 No. 1, pp. 18-27. https://doi.org/10.1080/09613210601036697

Wickens, C.D. and Carswell, C.M. (1995), "The proximity compatibility principle: Its psychological foundation and relevance to display design", Human Factors, Human Factors and Ergonomics Society, Inc., Vol. 37 No. 3, pp. 473-494. https://doi.org/10.1518/001872095779049408

Wood, R.E. (1986), "Task complexity: Definition of the construct", Organizational Behavior and Human Decision Processes, Vol. 37 No. 1, pp. 60-82. https://doi.org/10.1016/0749-5978(86)90044-0

Yoerger, M., Crowe, J. and Allen, J.A. (2015), "Participate or else!: The effect of participation in decisionmaking in meetings on employee engagement", Consulting Psychology Journal, Vol. 67 No. 1, pp. 65-80. https://doi.org/10.1037/cpb0000029 